\title{
Loss of phospholipase D2 impairs VEGF-induced angiogenesis
}

\author{
Chang Sup Lee ${ }^{1, \#}$, Jaewang Ghim ${ }^{1, \#}$, Parkyong Song ${ }^{1}$, Pann-Ghill Suh ${ }^{2}$ \& Sung Ho Ryu ${ }^{1, *}$ \\ ${ }^{1}$ Department of Life Sciences, Pohang University of Science and Technology (POSTECH), Pohang 37673, ${ }^{2}$ School of Life Sciences, Ulsan \\ National Institute of Science and Technology (UNIST), Ulsan 44919, Korea
}

\begin{abstract}
Vascular endothelial growth factor (VEGF) is a key mediator of angiogenesis and critical for normal embryonic development and repair of pathophysiological conditions in adults. Although phospholipase D (PLD) activity has been implicated in angiogenic processes, its role in VEGF signaling during angiogenesis in mammals is unclear. Here, we found that silencing of PLD2 by siRNA blocked VEGF-mediated signaling in immortalized human umbilical vein endothelial cells (iHUVECs). Also, VEGF-induced endothelial cell survival, proliferation, migration, and tube formation were inhibited by PLD2 silencing. Furthermore, while PId2-knockout mice exhibited normal development, loss of PLD2 inhibited VEGF-mediated ex vivo angiogenesis. These findings suggest that PLD2 functions as a key mediator in the VEGF-mediated angiogenic functions of endothelial cells. [BMB Reports 2016; 49(3): 191-196]
\end{abstract}

\section{INTRODUCTION}

VEGF signaling mediates an essential rate-limiting step of angiogenesis in endothelial cells $(1,2)$. From embryo to adult, angiogenesis plays key roles in development and basic life phenomena to maintain homeostasis of such vital functions as oxygen/nutrient supply and waste elimination $(3,4)$. Furthermore, an excess or shortage of angiogenesis affects various diseases, such as cancer, inflammatory disorders, obesity, asthma, diabetes, multiple sclerosis, ischemic heart disease and preeclampsia (3-5). While VEGF is crucial for both physiological and pathological angiogenesis, it is dramatically upregulated during pathological angiogenesis, such as tumor and diabetic retinopathy $(6,7)$. Therefore, discovery and characterization of the key factor that mediates VEGF signaling in endothelial cells is essential for understanding the roles of angiogenesis in path-

${ }^{*}$ Corresponding author. Tel: +82-54-279-2292; Fax: +82-54-2790645; E-mail: sungho@postech.ac.kr

${ }^{\#}$ These authors contributed equally to this work.

http://dx.doi.org/10.5483/BMBRep.2016.49.3.219

Received 23 October 2015, Revised 25 November 2015, Accepted 21 January 2016

Keywords: Angiogenesis, Aorta ring, Endothelial cells, Phospholipase D, Tube formation, VEGF ophysiological conditions.

Phospholipase D (PLD) is a phosphatidylcholine (PC)-hydrolyzing enzyme that generates phosphatidic acid (PA) and choline (8-11). In mammals, two PLD isozymes, PLD1 and PLD2, have been identified and have $\sim 50 \%$ sequence homology (8-11). PLD1 and PLD2 can be activated by a variety of upstream signals, such as growth factors, hormones, neurotransmitters, and phospholipids (8-11). PLD functions as a key amplifier/modulator between upstream signals and downstream mediators by producing the second messenger, PA. Recently, it has been reported that Src-dependent PLD1 is required for retinal neovascularization (12) and PLD1 is implicated in the formation of intersegmental blood vessels in zebrafish (13). Although both PLD1 and PLD2 have been implicated in numerous biological phenomena, including proliferation, vesicle trafficking, cytoskeletal rearrangement, and differentiation (8-11), it is unknown how each PLD isozyme (PLD1 and PLD2) mediates VEGF signaling and whether either (especially PLD2) plays a key role in VEGF-mediated endothelial cell functions and angiogenesis.

In this study, we investigated whether PLD1 and PLD2 mediate VEGF signaling in endothelial cells. We also determined whether PLD2 regulates the VEGF-mediated functions of endothelial cells at the cellular and ex vivo tissue levels. Therefore, this study provides new information regarding the regulation of VEGF-induced angiogenesis.

\section{RESULTS}

PLD isotype-specific signaling pathway in iHUVECs

PLD1 and PLD2 mediate not only similar functions, but also PLD isotype-specific functions (10, 11, 14-16). First, we investigated whether each PLD mediates isotype-specific angiogenic signaling in iHUVECs. After silencing of each PLD isotype, we assessed the activation of key mediators of angiogenesis in endothelial cells. We found that both PLD1 and PLD2 silencing blocked phosphorylation of S-6-kinase and ERK in iHUVECs (Fig. 1A). However, silencing of PLD2, but not PLD1, reduced Src phosphorylation. In addition, when we investigated the expression levels of genes related to angiogenesis in iHUVECs after silencing of PLD1 or PLD2 (Fig. 1B and 1C), silencing of PLD2, but not PLD1, significantly decreased the ANGPT1 mRNA level (Angiopoietin 1) (Fig. 1D). Furthermore, mRNA levels of KDR2 (VEGF receptor-2) and MMP2 (matrix metal- 
A

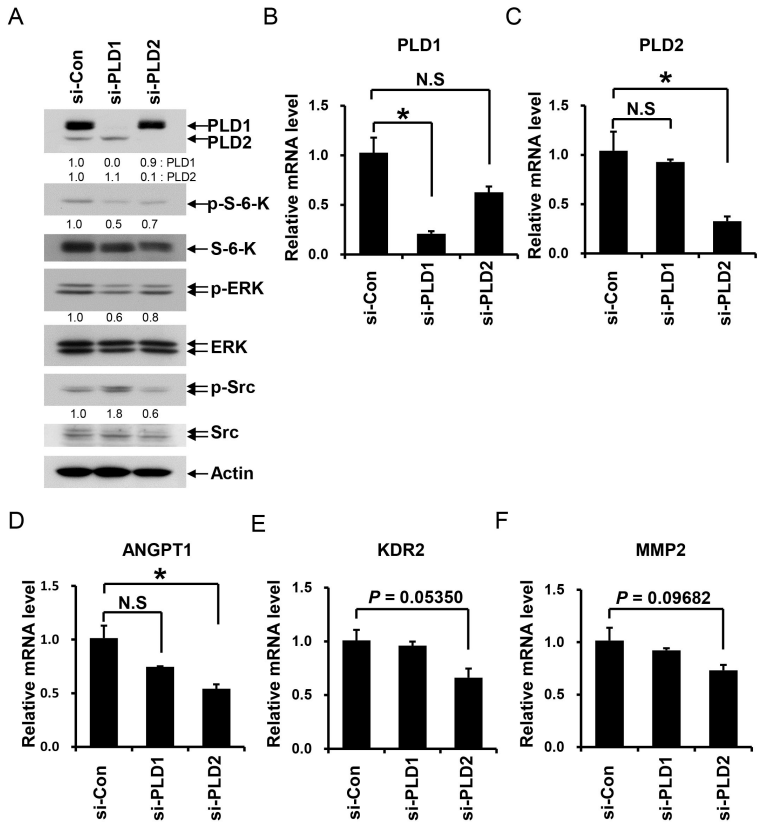

Fig. 1. Regulation of angiogenic signaling mediators and expression of angiogenesis-related genes by PLD1 and PLD2. (A) Following siRNA silencing of PLD1 or PLD2, iHUVECs were cultured in growth medium and then lysed with lysis buffer. The lysates were analyzed by Western blotting using the indicated antibodies. Bands were quantitatively analyzed by Image J (NIH) program. Quantitation of each band is shown below. (B-F) Following transfection of iHUVECs with siRNA against PLD1 or PLD2, total CDNA was isolated and the mRNA levels of specific genes (PLD1, PLD2, ANGPT1 (Angiopoietin 1), KDR2 (VEGF receptor-2) and MMP2) were measured by qPCR. Data are representative of at least two independent experiments. Error bars indicate S.D. ${ }^{*} P<0.05$. N. S.: not significant.

loproteinase2) were reduced by silencing of PLD2, but not PLD1 (Fig. 1E and 1F). These results suggest that the role in angiogenesis of PLD2 differs from that of PLD1.

\section{PLD2 is a key mediator of VEGF signaling in iHUVECs}

VEGF as a key mediator of angiogenesis activates multiple intracellular signaling pathways - including the phosphoinositide 3-kinase (PI 3-kinase), AKT, ERK, and Src pathways-in endothelial cells $(1,2)$. First, we investigated the roles of PLD isotypes in VEGF-mediated signaling after siRNA silencing of PLD1 or PLD2 in iHUVECs. We verified that VEGF increased the phosphorylation of S-6-kinase, ERK, and Src in a time-dependent manner (Fig. 2A), and a low VEGF concentration (2 $\mathrm{ng} / \mathrm{ml}$ ) induced the phosphorylation of S-6-kinase, ERK, and Src in iHUVECs (Fig. 2B). PLD1 or PLD2 silencing blocked the VEGF-induced phosphorylation of ERK, S-6-kinase, and Src in iHUVECs (Fig. 2C). In particular, p-ERK and p-S-6-kinase levels were inhibited more significantly by PLD2 silencing than PLD1 silencing. In the case of Src, silencing of PLD2, but not PLD1, inhibited Src phosphorylation (Fig. 2C). These results
A
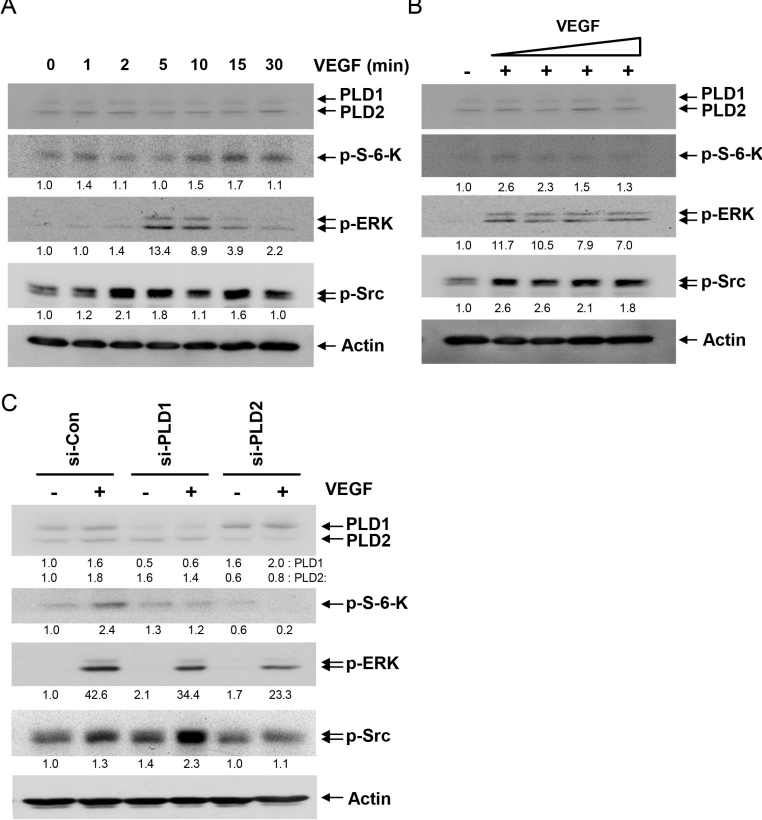

Fig. 2. PLD2 mediates VEGF signaling in iHUVECs. (A) iHUVECs were starved for $24 \mathrm{~h}$ and then treated with VEGF $(10 \mathrm{ng} / \mathrm{ml})$ for the indicated times. (B) iHUVECs were starved for $24 \mathrm{~h}$ and then treated with various VEGF doses $(0,2,5,10$, or $40 \mathrm{ng} / \mathrm{ml})$ for $10 \mathrm{~min}$. (C) Following siRNA silencing of PLD1 or PLD2, iHUVECs were starved for $24 \mathrm{~h}$ and then treated with VEGF $(10 \mathrm{ng} / \mathrm{ml})$ for $10 \mathrm{~min}$. The lysates were analyzed via SDS-PAGE and Western blotting using the indicated antibodies. Data are representative of at least two independent experiments. Bands were quantitatively analyzed by Image $\mathrm{J}(\mathrm{NIH})$ program. Quantitation of each band is shown below.

suggest that PLD2 is essential for VEGF signaling in iHUVECs and that the VEGF-PLD2 pathway differs from the VEGF-PLD1 pathway.

\section{PLD2 deficiency leads to defects in VEGF-induced cell survival, proliferation, migration, and tube formation in iHUVECS}

VEGF signaling mediates various endothelial cell functionssuch as migration, proliferation, and survival-via multiple intracellular signaling pathways including the Src, phosphoinositide 3-kinase (PI 3-kinase), AKT, and ERK pathways (1, 2, 4). Thus, we investigated the effect of PLD2 on the role of VEGF in the survival, proliferation, migration, and tube formation in iHUVECs. As shown in Fig. 3A and 3B, VEGF enhanced the survival and proliferation of iHUVECs. However, silencing of PLD2 blocked VEGF-induced cell survival and proliferation (Fig. 3A and 3B). Moreover, VEGF-induced migration and tube formation (in vitro angiogenesis) were also inhibited by silencing of PLD2 (Fig. 3C and 3D). Tube formation assay of in vitro angiogenesis has been reported to represent cell adhesion, migration, protease activation, and tubule formation in endothe- 
A

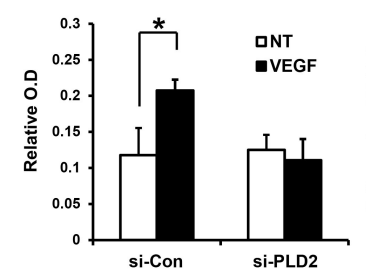

C

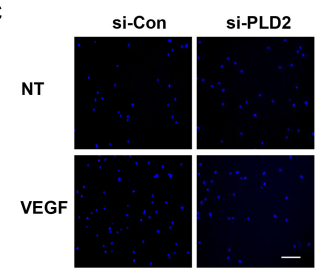

D

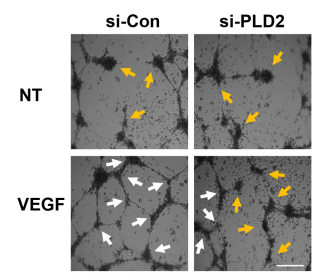

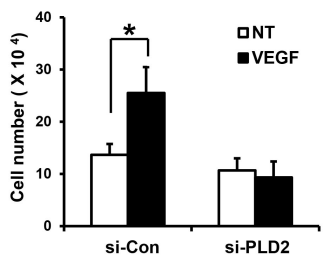

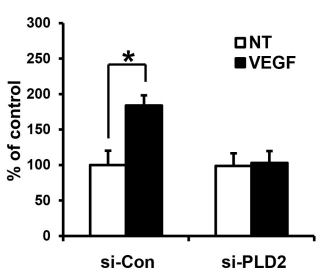

Fig. 3. PLD2 plays key roles in VEGF-induced iHUVEC survival, proliferation, migration and tube formation. (A and B) Following siRNA silencing of PLD2, iHUVECs were starved for $24 \mathrm{~h}$ and then treated with VEGF $(20 \mathrm{ng} / \mathrm{ml})$ for $48 \mathrm{~h}$. Cell viability was measured by MTT assay. Cells were enumerated using a hemocytometer. (C) Following siRNA silencing of PLD2, iHUVECs were starved for $24 \mathrm{~h}$ and then detached. The detached cells were replated on the upper well of a chemotaxis chamber. Chemotactic migration was measured in the absence or presence of VEGF $(10 \mathrm{ng} / \mathrm{ml})$. Scale bar, 100 $\mu \mathrm{m}$. (D) Following siRNA silencing of PLD2, iHUVECs were starved for $24 \mathrm{~h}$ and then detached. Cells were replated on matrigel-coated dishes in the absence or presence of VEGF $(40 \mathrm{ng} / \mathrm{ml})$. The white arrows indicate intact branching points of network. The yellow arrows indicate points where the branching network failed to occur. Scale bar, $250 \mu \mathrm{m}$. Data are representative of at least two independent experiments. Error bars indicate S.D. ${ }^{* P}<0.05$.

lial cells (17). Multiple sequential steps-such as cell adhesion, survival, proliferation, and migration, protease activation, and tubule formation -in endothelial cells are critical for VEGF-induced angiogenesis in vivo $(1,2,4,5)$. Therefore, these data imply that PLD2 contributes to angiogenesis through multiple processes such as VEGF-induced cell survival, proliferation, migration, and tubule formation.

\section{PLD2 plays a role in VEGF-induced sprouting from aorta}

Our in vitro data suggest that PLD2 might also mediate VEGF angiogenesis in vivo. We generated Pld2-knockout mice to reveal the roles of PLD2 in angiogenesis (18). First, we investigated the effect of PLD2 deficiency on tissue and organ development. As shown in Fig. 4A, at day E15.5, when fetal organogenesis was completed (19), we found that the genotypes of fetuses exhibited a Mendelian ratio, as expected.

A

\begin{tabular}{|c|c|c|c|c|c|}
\hline Age & Litter & $P l d 2^{+/+}$ & $P I d 2^{+/-}$ & PId $2^{-1}$ & Total \\
\hline E15.5 & 8 & 20 & 50 & 21 & 91 \\
\hline \multicolumn{2}{|c|}{ Total (\%): observed } & $22.0 \%$ & $54.9 \%$ & $23.1 \%$ & \\
\hline \multicolumn{2}{|c|}{ Total (\%): expected } & $22(25 \%)$ & $45(50 \%)$ & $22(25 \%)$ & \\
\hline Age & Litter & $\mathrm{Pld}^{+/+}$ & $\mathrm{Pld}^{+\mathbf{H}^{+}}$ & Pld2'- & Total \\
\hline Po & 12 & 19 & 43 & 21 & 83 \\
\hline \multicolumn{2}{|c|}{ Total (\%): observed } & $22.9 \%$ & $51.8 \%$ & $26.0 \%$ & \\
\hline \multicolumn{2}{|c|}{ Total (\%): expected } & $20.5(25 \%)$ & $42(50 \%)$ & $20.5(25 \%)$ & \\
\hline
\end{tabular}

B

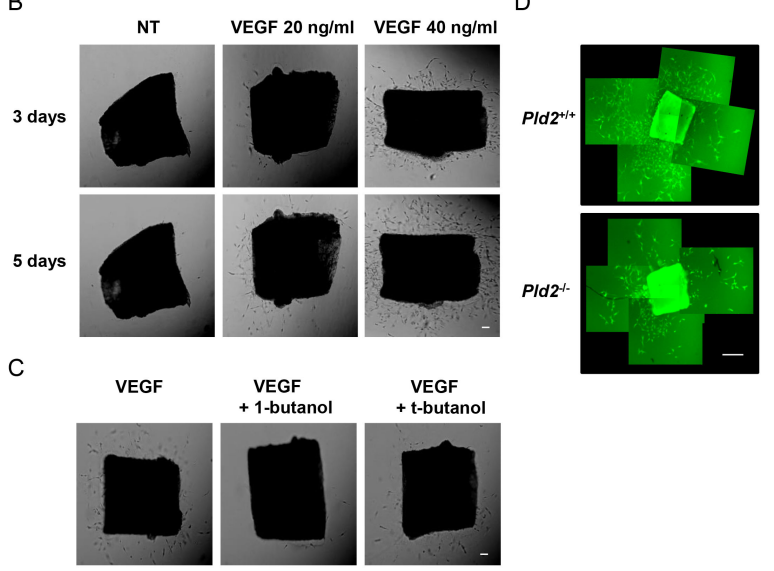

Fig. 4. Aortas of Pld2-knockout mice show reduced VEGF-induced sprouting. (A) Mouse embryos and pups were genotyped at days E15.5 and P0, respectively. (B) Aortas were isolated from control and Pld2-knockout mice and implanted on matrigel. Aortas were treated with the indicated VEGF dose for the indicated time. Scale bar, $100 \mu \mathrm{m}$. (C) Aortas were treated with VEGF $(20 \mathrm{ng} / \mathrm{ml})$ in the absence or presence of 1-butanol or t-butanol. Scale bar, $100 \mu \mathrm{m}$. (D) Aortas from control and Pld2-knockout mice were treated with VEGF $(40 \mathrm{ng} / \mathrm{ml})$ for 5 days. Scale bar, $500 \mu \mathrm{m}$. Data are representative of at least two independent experiments.

Furthermore, mouse pups at P0 were born with a Mendelian ratio and showed normal development. Next, we investigated the effect of PLD2 deficiency on VEGF-induced angiogenesis. VEGF induces sprouting from mouse aorta in an ex vivo aortic ring assay, which is a more physiologically relevant model of angiogenesis and preserves certain key features of in vivo angiogenesis, which is mediated by various cell types (endothelial cells, macrophages, pericytes, smooth muscle cells and fibroblasts) $(20,21)$. VEGF-induced endothelial cell (EC) sprouting from the aorta occurred in a dose- and time-dependent manner (Fig. 4B). 1-Butanol, a blocker of PA generation from PLD, inhibited VEGF-induced EC sprouting from the aorta. However, t-butanol, as a control treatment, did not inhibit VEGF-induced EC sprouting (Fig. 4C). These results indicate that PLD activity is important for VEGF-mediated EC sprouting. When we investigated the involvement of PLD2 in ex vivo angiogenesis (aortic ring assay) using Pld2-knockout mice, we also found that PLD2 deficiency inhibited VEGF-induced aortic sprouting (Fig. 4D). These data suggest that PLD2 is involved 
in VEGF-mediated angiogenesis, although the cell-type specific roles of PLD2 in the aortic ring, which comprises multiple cell types, are unclear.

\section{DISCUSSION}

Here, we elucidated that PLD2 functioned as a key modulator of VEGF signaling pathways (ERK, S-6-kinase, and Src) in iHUVECs. This function of PLD2 was essential for survival, proliferation, migration, and tube formation of endothelial cells, which mediate new vessel formation or angiogenesis from existing vessels. Also, using an ex vivo Pld2-knockout mouse model, we revealed PLD2 to be an essential mediator of VEGF-mediated angiogenesis. Therefore, this work enhances our understanding of the intracellular mediators of VEGFinduced angiogenesis.

VEGF binds to and activates VEGF receptor-2 (VEGFR-2) on endothelial cells. VEGF binding induces the tyrosine phosphorylation of VEGFR-2 at multiple sites (Y951, Y1054, Y1059, Y1175, and Y1214) (2, 22-24). These tyrosine-phosphorylated sites recruit several downstream molecules - such as TSAd, Shb, Sck, and PLC $\gamma$-which mediate multiple functions-such as migration (Src or FAK pathway), survival (PI3 kinase-AKTS-6-kinase pathway), proliferation (ERK pathway) and vascular permeability (eNOS pathway) — of endothelial cells (2, 22-33). Our results indicate that PLD2 silencing inhibits VEGF-induced phosphorylation of S-6-kinase, ERK, and Src in iHUVECs (Fig. $2 \mathrm{C})$. Thus PLD2 may play a role as a key regulator of these key mediators of angiogenesis (ERK, S-6-kinase, and Src).

Recently, it has been reported that Src functions upstream of PLD1 in VEGF-mediated signaling of human retinal microvascular endothelial cells (HRMVECs) (12). However, we found that PLD1 functioned upstream of Src in VEGF signaling and PLD1 silencing resulted in a slight increase in VEGF-induced phosphorylation of Src compared to control silencing, while PLD2 silencing resulted in a reduction in VEGF-mediated Src phosphorylation in iHUVECs (Fig. 2C). This result is in agreement with a previous report that silencing of PLD1 increased the p-Src and p-Pyk2 levels, but PLD2 silencing had no significant effect (a slight reduction) on Src and Pyk2 phosphorylation in HeLa cells (14). Furthermore, mRNA levels of angiogenic related-genes were differently regulated by silencing of each PLD isotype in iHUVECs (Fig. 1D-F). Although whether these results are cell-type or signal specific is unclear, they suggest that PLD2 mediates VEGF signaling in a manner different from PLD1.

VEGF-neutralizing antibodies and VEGFR inhibitors have been used to target pathological angiogenesis, such as tumor angiogenesis $(2,34)$. However, as shown in knockout-mouse studies of VEGF and VEGFR, deficiency of these molecules caused embryonic lethality and defects in organ development due to failure of angiogenesis (2, 34-39). These data indicate that molecules that target VEGF and VEGFR may affect physiological angiogenesis. Here, we found that Pld2-knockout mice were born normally (a Mendelian frequency) (Fig. 4A). Therefore, PLD2 did not critically affect normal development and physiological angiogenesis. However, PLD2 deficiency inhibited VEGF (40 ng/ml)-mediated ex vivo angiogenesis (Fig. $4 \mathrm{D})$. In mouse and human, the physiological level of VEGF is $\sim 0.065 \mathrm{ng} / \mathrm{ml}$ and $0.137 \mathrm{ng} / \mathrm{ml}$, respectively $(40,41)$. In pathological tissue such as tumors, the VEGF level is $10-200$ fold higher than that in normal tissue (42). Taken together, these reports and our findings suggest that PLD2 plays a key role in a high local concentration of VEGF, such as in pathological conditions. Therefore, we suggest that angiogenic targeting of PLD2, which is a key downstream molecule of VEGF signaling, may facilitate development of a drug targeted specifically at pathological conditions, while not negatively affecting the overall physiological condition.

\section{MATERIALS AND METHODS}

\section{Animal}

All mice were kept in an animal facility at the Pohang University of Science and Technology (POSTECH). Animal experiments were performed according to the institutional guidelines of POSTECH. To generate whole-body Pld2-knockout (KO) mice, Pld2 $2^{\mathrm{fl} / \mathrm{fl}}$ mice were crossed with Protamine-Cre (Jackson Laboratory) and Pld2 KO mice then maintained without the Cre allele. Pld2 KO mice were backcrossed with $\mathrm{C} 57 \mathrm{BL} / 6$ for at least 14 generations.

\section{Cell culture and transfection}

Immortalized iHUVECs were maintained at $37^{\circ} \mathrm{C}$ in $5 \% \mathrm{CO}_{2}$ in M199 medium supplemented with 10\% FBS (Lonza), $5 \mathrm{mM}$ L-glutamine (Life Technologies), endothelial cell growth supplement (BD Biosciences), penicillin (100 units/ml) and streptomycin $(0.1 \mathrm{mg} / \mathrm{ml})$. For PLD silencing, iHUVECs were transfected with control siRNA (luciferase GL2 duplex; Dharmacon Research) or PLD1 and/or PLD2 siRNA (20 nM duplex) using Lipofectamine (Invitrogen) according to the manufacturer's instruction and then cultured for $48 \mathrm{~h}$.

\section{Westem blotting}

Samples were lysed in lysis buffer $(20 \mathrm{mM}$ Tris/pH 8.0, 150 $\mathrm{mM} \mathrm{NaCl}, 1 \mathrm{mM}$ EDTA, $0.1 \%$ SDS, $1.0 \%$ Triton X-100, $1.0 \%$ sodium deoxycholate, and protease inhibitor cocktail (Sigma)). After centrifugation at $10,000 \times \mathrm{g}$ for $10 \mathrm{~min}$ at $4^{\circ} \mathrm{C}$, the protein concentration of supernatants was determined by Bradford assay. Laemmli sample buffer (5X) was added to the samples, which were then separated by SDS-PAGE and transferred onto nitrocellulose membranes $(0.2 \mu \mathrm{m}$ pore size). The membranes were blocked in blocking solution ( $5 \%$ non-fat milk in TTBS), then incubated with primary antibodies for $4 \mathrm{~h}$ or overnight at room temperature, followed by incubation with secondary antibodies (horseradish peroxidase (HRP)-conjugated) for $1 \mathrm{~h}$. Blots were detected using an enhanced chemiluminescence kit. 


\section{Quantitative PCR (qPCR)}

Total RNA from iHUVECs was isolated using TRIzol reagent, and cDNAs were generated from $1 \mu \mathrm{g}$ of total RNA. qPCR was performed with HotStart-IT SYBR ${ }^{\mathbb{R}}$ Green and a Bio-Rad iCycler iQ instrument using specific primers (PLD1 (F:TGCGTCTACA TCCCAACATAAA, R:AGGTCAATCCCTCCCACAA), PLD2 (F: GCTCCСCTTCACACTTCCAG, R:GCAGGTAGGCATTGAGGA TG), ANGPT1 (F:GACAGCAGGAAAACAGAGCAG, R: CACA AGCATCAAACCACCATC), KDR2 (F:CAGAGTTGGTGGAACA TTTGG, R:CAGGAAACAGGTGAGGTAGGC), MMP2 (F:TACC CCAAGCCACTGACCA, R:ACGACGGCATCCAGGTTAT)). The relative $\mathrm{mRNA}$ quantities were calculated by the comparative Ct method after normalization to GAPDH.

\section{MTT assay}

MTT assay were performed as previously described, with some modifications (43). Cells were plated in 12-well plates and transfected with control or PLD2 siRNA using Lipofectamine (Invitrogen). After culturing for a further 2 days, cells were starved for $24 \mathrm{~h}$ and then treated with VEGF $(20 \mathrm{ng} / \mathrm{ml})$ for 48 h. Tetrazolium dye, MTT $(0.5 \mathrm{mg} / \mathrm{ml})$ was added to each well and the plates were incubated for $4 \mathrm{~h}$ at $37^{\circ} \mathrm{C}$. Remaining MTT was removed and DMSO was added to each well to dissolve formazan crystals. Absorbance at $540 \mathrm{~nm}$ was measured using a spectrophotometer.

\section{Proliferation}

Cells were plated in six-well plates and transfected with control or PLD2 siRNA using Lipofectamine (Invitrogen). After being cultured for a further 2 days, cells were starved for $24 \mathrm{~h}$ and then treated with VEGF $(20 \mathrm{ng} / \mathrm{ml})$ for $48 \mathrm{~h}$. For enumeration, cells were detached with trypsin and then counted using a hemocytometer.

\begin{abstract}
Migration
Cell migration was measured using a modified Boyden chemotaxis chamber (NeuroProbe Inc.) as previously described, with some modifications (44). Polycarbonate membranes (8 $\mu \mathrm{m}$ pore size) were coated with collagen $(20 \mu \mathrm{g}$, Sigma). Culture medium containing VEGF $(10 \mathrm{ng} / \mathrm{ml})$ was added to the bottom wells of the chamber. After starvation for $24 \mathrm{~h}$, cells were detached and then resuspended in serum-free medium. The cells in the upper chamber were plated at a density of $1 \times 10^{4}$ cells. The chambers were incubated at $37^{\circ} \mathrm{C}$ for $3 \mathrm{~h}$. Non-migrating cells in the upper chamber were removed from the membrane, and migrated cells were stained with Hoechst dye (Sigma).
\end{abstract}

\section{Tube formation}

After starvation for $24 \mathrm{~h}$, iHUVECs $\left(1 \times 10^{5}\right.$ per well $)$ were plated into 48-well culture plates coated with growth factor-reduced matrigel (BD Biosciences) and then incubated for $9 \mathrm{~h}$ at $37^{\circ} \mathrm{C}$ in M199 medium containing VEGF (40 ng/ml). Images were obtained using a Zeiss Axiovert 135 microscope.

\section{Aortic ring assay}

After euthanasia, mice were perfused with cold PBS $(10 \mathrm{ml})$ containing $1 \%$ FBS. The aortic ring was excised from each mouse and cut into $1 \mathrm{~mm}$ lengths. The aortic rings were implanted in growth factor reduced Matrigel (BD Biosciences) and incubated with EBM-2 medium supplemented with VEGF ( 20 or $40 \mathrm{ng} / \mathrm{ml}$ ) for 5 days.

\section{Statistical analysis}

The data are presented as means \pm SD. Statistical analyses were performed using Student's t-test. ${ }^{*} \mathrm{P}<0.05$.

\section{ACKNOWLEDGEMENTS}

This work was supported by the National Research Foundation of Korea (NRF) grant funded by the Korea government (MEST) (No. NRF-2015R1A2A1A13001834).

\section{REFERENCES}

1. Ferrara N, Gerber HP and LeCouter J (2003) The biology of VEGF and its receptors. Nat Med 9, 669-676

2. Olsson AK, Dimberg A, Kreuger J and Claesson-Welsh L (2006) VEGF receptor signalling - in control of vascular function. Nat Rev Mol Cell Biol 7, 359-371

3. Carmeliet P and Jain RK (2011) Molecular mechanisms and clinical applications of angiogenesis. Nature 473, 298-307

4. Carmeliet $P$ (2005) Angiogenesis in life, disease and medicine. Nature 438, 932-936

5. Ribatti D, Nico B and Crivellato E (2009) Morphological and molecular aspects of physiological vascular morphogenesis. Angiogenesis 12, 101-111

6. Behl T and Kotwani A (2015) Exploring the various aspects of the pathological role of vascular endothelial growth factor (VEGF) in diabetic retinopathy. Pharmacol Res 99, 137-148

7. Hoeben A, Landuyt B, Highley MS, Wildiers H, Van Oosterom AT and De Bruijn EA (2004) Vascular endothelial growth factor and angiogenesis. Pharmacol Rev 56, 549-580

8. Frohman MA (2015) The phospholipase D superfamily as therapeutic targets. Trends Pharmacol Sci 36, 137-144

9. Gomez-Cambronero J (2014) Phospholipase D in cell signaling: from a myriad of cell functions to cancer growth and metastasis. J Biol Chem 289, 22557-22566

10. Jang JH, Lee CS, Hwang D and Ryu SH (2012) Understanding of the roles of phospholipase D and phosphatidic acid through their binding partners. Prog Lipid Res 51, 71-81

11. Nelson RK and Frohman MA (2015) Physiological and Pathophysiological roles for Phospholipase D. J Lipid Res 56, 2229-2237

12. Zhang Q, Wang D, Kundumani-Sridharan V et al (2010) PLD1-dependent PKCgamma activation downstream to Src is essential for the development of pathologic retinal neovascularization. Blood 116, 1377-1385

13. Zeng XX, Zheng X, Xiang Y et al (2009) Phospholipase D1 
is required for angiogenesis of intersegmental blood vessels in zebrafish. Dev Biol 328, 363-376

14. Kim JH, Kim HW, Jeon H, Suh PG and Ryu SH (2006) Phospholipase D1 regulates cell migration in a lipase activity-independent manner. J Biol Chem 281, 15747-15756

15. Jenkins GM and Frohman MA (2005) Phospholipase D: a lipid centric review. Cell Mol Life Sci 62, 2305-2316

16. Cockcroft $S$ (2001) Signalling roles of mammalian phospholipase D1 and D2. Cell Mol Life Sci 58, 1674-1687

17. Arnaoutova I and Kleinman HK (2010) In vitro angiogenesis: endothelial cell tube formation on gelled basement membrane extract. Nat Protoc 5, 628-635

18. Ghim J, Moon JS, Lee CS et al (2014) Endothelial deletion of phospholipase D2 reduces hypoxic response and pathological angiogenesis. Arterioscler Thromb Vasc Biol 34, 1697-1703

19. Hedrich HJ and Bullock GR (2004) The laboratory mouse, Elsevier Academic Press, Amsterdam; Boston

20. Aplin AC, Fogel E, Zorzi P and Nicosia RF (2008) The aortic ring model of angiogenesis. Methods Enzymol 443, 119-136

21. Baker M, Robinson SD, Lechertier T et al (2012) Use of the mouse aortic ring assay to study angiogenesis. Nat Protoc 7, 89-104

22. Dougher M and Terman BI (1999) Autophosphorylation of $\mathrm{KDR}$ in the kinase domain is required for maximal VEGF-stimulated kinase activity and receptor internalization. Oncogene 18, 1619-1627

23. Takahashi T, Yamaguchi S, Chida K and Shibuya M (2001) A single autophosphorylation site on KDR/Flk-1 is essential for VEGF-A-dependent activation of PLC-gamma and DNA synthesis in vascular endothelial cells. EMBO J 20, 2768-2778

24. Holmqvist K, Cross MJ, Rolny C et al (2004) The adaptor protein shb binds to tyrosine 1175 in vascular endothelial growth factor (VEGF) receptor-2 and regulates VEGF-dependent cellular migration. J Biol Chem 279, 22267-22275

25. Dayanir V, Meyer RD, Lashkari K and Rahimi N (2001) Identification of tyrosine residues in vascular endothelial growth factor receptor-2/FLK-1 involved in activation of phosphatidylinositol 3-kinase and cell proliferation. J Biol Chem 276, 17686-17692

26. Fujio $\mathrm{Y}$ and Walsh K (1999) Akt mediates cytoprotection of endothelial cells by vascular endothelial growth factor in an anchorage-dependent manner. J Biol Chem 274, 16349-16354

27. Warner AJ, Lopez-Dee J, Knight EL, Feramisco JR and Prigent SA (2000) The Shc-related adaptor protein, Sck, forms a complex with the vascular-endothelial-growthfactor receptor KDR in transfected cells. Biochem J 347, 501-509

28. Takahashi T, Ueno H and Shibuya M (1999) VEGF activates protein kinase C-dependent, but Ras-independent Raf-MEK-MAP kinase pathway for DNA synthesis in primary endothelial cells. Oncogene 18, 2221-2230

29. Kroll J and Waltenberger J (1997) The vascular endothelial growth factor receptor KDR activates multiple signal transduction pathways in porcine aortic endothelial cells. J Biol
Chem 272, 32521-32527

30. Matsumoto T, Bohman S, Dixelius J et al (2005) VEGF receptor-2 Y951 signaling and a role for the adapter molecule TSAd in tumor angiogenesis. EMBO J 24, 2342-2353

31. Zeng H, Sanyal S and Mukhopadhyay D (2001) Tyrosine residues 951 and 1059 of vascular endothelial growth factor receptor-2 (KDR) are essential for vascular permeability factor/vascular endothelial growth factor-induced endothelium migration and proliferation, respectively. J Biol Chem 276, 32714-32719

32. Abedi $\mathrm{H}$ and Zachary I (1997) Vascular endothelial growth factor stimulates tyrosine phosphorylation and recruitment to new focal adhesions of focal adhesion kinase and paxillin in endothelial cells. J Biol Chem 272, 15442-15451

33. Fulton D, Gratton JP, McCabe TJ et al (1999) Regulation of endothelium-derived nitric oxide production by the protein kinase Akt. Nature 399, 597-601

34. Schlaeppi JM and Wood JM (1999) Targeting vascular endothelial growth factor (VEGF) for anti-tumor therapy, by anti-VEGF neutralizing monoclonal antibodies or by VEGF receptor tyrosine-kinase inhibitors. Cancer Metastasis Rev $18,473-481$

35. Carmeliet P, Ferreira V, Breier G et al (1996) Abnormal blood vessel development and lethality in embryos lacking a single VEGF allele. Nature 380, 435-439

36. Ferrara N, Carver-Moore K, Chen H et al (1996) Heterozygous embryonic lethality induced by targeted inactivation of the VEGF gene. Nature 380, 439-442

37. Fong $\mathrm{GH}$, Rossant J, Gertsenstein $M$ and Breitman $M L$ (1995) Role of the Flt-1 receptor tyrosine kinase in regulating the assembly of vascular endothelium. Nature 376, 66-70

38. Shalaby F, Rossant J, Yamaguchi TP et al (1995) Failure of blood-island formation and vasculogenesis in Flk-1-deficient mice. Nature 376, 62-66

39. Dumont DJ, Jussila L, Taipale J et al (1998) Cardiovascular failure in mouse embryos deficient in VEGF receptor-3. Science 282, 946-949

40. Sugimoto $H$, Hamano Y, Charytan D et al (2003) Neutralization of circulating vascular endothelial growth factor (VEGF) by anti-VEGF antibodies and soluble VEGF receptor 1 (sFlt-1) induces proteinuria. J Biol Chem 278, 12605-12608

41. Czarkowska-Paczek B, Bartlomiejczyk I and Przybylski J (2006) The serum levels of growth factors: PDGF, TGF-beta and VEGF are increased after strenuous physical exercise. J Physiol Pharmacol 57, 189-197

42. Takano S, Yoshii Y, Kondo S et al (1996) Concentration of vascular endothelial growth factor in the serum and tumor tissue of brain tumor patients. Cancer Res 56, 2185-2190

43. Kim GY, Park SY, Jo A et al (2015) Gecko proteins induce the apoptosis of bladder cancer 5637 cells by inhibiting Akt and activating the intrinsic caspase cascade. BMB Rep 48, 531-536

44. Qin JF, Jin FJ, Li N et al (2015) Adrenergic receptor $\beta 2$ activation by stress promotes breast cancer progression through macrophages M2 polarization in tumor microenvironment. BMB Rep 48, 295-300 